[4] W. Pogorzelski, Problème aux limites de Poincaré généralisé, Ann. Pol.

2 (1955), p. $257-270$

[5] J. Wolska-Bochenek, Un problème aux limites à dérivée tangentielle pour l'équation du type elliptique, Ann. Pol. Math. 4 (1958), p. 275-287.

[6] J. Schauder, Der Fixpunletsatz in Frunletionalraümen, Studia Math. 2 (1930), p. $171-180$.

\section{About the extremal spiral schlicht functions}

\author{
by J. ZAMORsKI (Wrocław)
}

Let $S$ denote a class of regular schlicht functions with the expansion

$$
f(z)=z+a_{2} z^{2}+\ldots
$$

for $|z|<1$, and let $\Sigma$ denote a class of meromorphic schlicht functions with the development

$$
F(z)=\frac{1}{z}+b_{0}+b_{1} z+\ldots
$$

for $0<|z|<1$. L. Spaček in his paper [3] proves that each function

$$
f(z)=z \exp \left\{\frac{1}{1-a i} \int_{0}^{z} \frac{p(z)-1}{z} d z\right\}
$$

where $p(z)=1+\alpha_{1} z+a_{2} z^{2}+\ldots,|z|<1$, re $p(z) \geqslant 0$ and $a$ is any reail number, belongs to the class $S$. Further Spaček proves that for these functions the hypothesis of Bieberbach is true. Of course, similarly

$$
F(z)=\frac{1}{z} \exp \left\{-\frac{1}{1-a i} \int_{0}^{z} \frac{p(z)-1}{z} d z\right\}
$$

belongs to the class $\Sigma$.

Let $S_{a}$ denote the class of functions defined by formula (3), and let $\Sigma_{\alpha}$ denote the class of functions defined by formula (4). We can easily see that for $a=0$ class $S_{a}$ becomes class $S^{*}$ of all functions starlike for the origin of the system of coordinates. Similarly for the class $\Sigma_{\alpha}$.

Let $W_{n}^{a}$ denote the $n$-th region of the coefficients of functions of the class $S_{a}$. We can easily see that it is a $2 n-2$ dimensional domain, closed, connected and bounded, including the origin of the system of coordinates. Namely from (3) we have

$$
\frac{z f^{\prime}(z)}{f(z)}=1+\varrho[p(z)-1]
$$


where $\varrho=1 /(1-a i)$ and re $p(z) \geqslant 0$. From (5) we obtain a connexion of the coefficients of the function with a positive real part and the coefficients of a function belonging to the class $S_{a}$,

(6) $\quad \frac{1-k}{\varrho} a_{k}+\alpha_{1} a_{k-1}+\ldots+\alpha_{k-2} a_{2}+\alpha_{k-1}=0, \quad k=2,3, \ldots$

Hence of $\operatorname{course}$

and

$$
a_{k}=\frac{(-1)^{k}}{(k-1) !} \varrho^{k-1}\left|\begin{array}{cccc}
\alpha_{1} & \alpha_{2} & \ldots & \alpha_{k-1} \\
\frac{1-k}{\varrho} & \alpha_{1} & \ldots & \alpha_{k-2} \\
0 & \frac{2-k}{\varrho} & \ldots & \alpha_{k-3} \\
\ldots & \ldots & \ldots & \ldots \\
0 & 0 & \ldots & \alpha_{1}
\end{array}\right|
$$

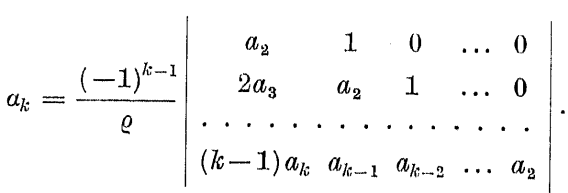

Formulae (7) and (8) give homeomorphism of the $n$-th region of variability of functions belonging to the class $S_{a}$ and the $n-1$ variability region of the coefficients of functions with a positive real part (see Carathéodory [1]), and this proves the above properties for the region $W_{n}^{a}$. The $n$-th variability region of coefficients of a function of the class $\Sigma_{a}$, denoted as $V_{n}^{a}$, has analogous properties.

Now we are going to define the functional $H_{n}(f)$ for functions of the class $S_{a}$. Namely let $H_{n}\left(x_{2}, \ldots, x_{n} ; y_{2}, \ldots, y_{n}\right)$ be a real function of $2 n-2$ real variables, defined in the region comprising the $n$-th region of variability $W_{n}^{a}$ and such that

$$
\sum_{k=2}^{n}\left[\left(\frac{\partial H_{n}}{\partial x_{k}}\right)^{2}+\left(\frac{\partial H_{n}}{\partial y_{k}}\right)^{2}\right] \neq 0
$$

for all points of the region $W_{n}^{a}$. This function will be considered as a functional defined for the functions of the class $S_{a}$, namely

$$
H_{n}(f)=H_{n}\left(x_{2}, \ldots, x_{n} ; y_{2}, \ldots, y_{n}\right), \quad f(z)=z+a_{2} z^{2}+\ldots, \quad a_{k}=x_{k}+i y_{k} .
$$

We are going to denote analogically the functional $E_{n}(F)$ for the functions of the class $\Sigma_{a}$. Now we shall study the extrema of the functionals $H_{n}(f)$ and $E_{n}\left(F^{\prime}\right)$.
THEOREM 1. The function of the class $S_{a}$ for which the functional $H_{n}(f)$ obtains its extremal value always has the following form

$$
\begin{gathered}
f(z)=z\left[\prod_{k=1}^{n-1}\left(1-\sigma_{k} z\right)^{-\beta_{k}}\right]^{1 /(1-a i)}, \\
\sum_{k=1}^{n-1} \beta_{k}=2, \quad \beta_{k}>0, \quad\left|\sigma_{k}\right|=1 .
\end{gathered}
$$

THEOREM 2. The function of the class $\Sigma_{a}$ for which the functional $E_{n}(F)$ obtains its extremal value always has the following form

$$
\begin{aligned}
F(z) & =\frac{1}{z}\left[\prod_{k=1}^{n+1}\left(1-\sigma_{k} z\right)^{\beta_{k}}\right]^{1 /(1-a i)}, \\
\sum_{k=1}^{n+1} \beta_{k} & =2, \quad \beta_{k}>0, \quad\left|\sigma_{k}\right|=1 .
\end{aligned}
$$

In a particular case, when we put $a=0$, we obtain the known theorems concerning starlike functions (see [4]).

Proof of theorem 1. Formulae (7) and (8) show that each point of the n-th variability region of the functions of the class $S_{a}$ corresponds continuously to one and only one point of the $n-1$ variability region of the functions with a positive real part, and vice versa. Hence the points of the boundary of one region correspond to the points of the boundary of the other and vice versa. From the assumptions on functional $H_{n}(f)$ infer this functional has its extremal value on the boundary of the region $W_{n}^{a}$. The extremal function corresponding to the boundary point of the region $W_{n}^{a}$ corresponds, according to formula (3), to the function $p(z)$, which is a boundary function of the $n-1$ variability region of functions with a positive real part-on the basis of the above correspondence.

By Carathéodory's theorem [1] this function must be of following form

$$
\begin{aligned}
p(z) & =1+\sum_{k=1}^{n-1} \beta_{k} \frac{\sigma_{k} z}{1-\sigma_{k} z}, \\
\sum_{k=1}^{n-1} \beta_{k} & =2, \quad \beta_{k}>0, \quad\left|\sigma_{k}\right|=1
\end{aligned}
$$


or

$$
f(z)=z \exp \left\{\frac{1}{1-a i} \int_{0}^{z} \sum_{k=1}^{n-1} \frac{\beta_{k} \sigma_{k}}{1-\sigma_{k} z} d z\right\}=z\left[\prod_{k=1}^{n-1}\left(1-\sigma_{k} z\right)^{-\beta_{k}}\right]^{1 /(1 \cdots a i)}
$$

which proves the theorem.

The proof of theorem 2 is analogical.

THEOREM 3. The parameters defining the function of the class $S_{a}$ extremal for the functional $H_{n}(f)$ satisfy the following system of equations:

$$
\boldsymbol{A}_{n-1} \sigma_{k}^{2(n-1)}+\ldots+A_{1} \sigma_{l}^{n}+\lambda \sigma_{l c}^{n-1}+\bar{A}_{1} \sigma_{l}^{n-2}+\ldots+\bar{A}_{n-1}=0,
$$

$2(n-1) A_{n-1} \sigma_{k}^{2 n-3}+\ldots+n A_{1} \sigma^{n--1}+(n-1) \lambda \sigma_{k}^{n-2}+$

$$
\begin{array}{r}
+(n-2) \bar{A}_{1} \sigma_{k}^{n-3}+\ldots+\bar{A}_{n-2}=0, \\
A_{j}=\frac{1}{1-a i} \cdot \frac{1}{j} \cdot \sum_{p=1}^{n-j} a_{p}\left\{\frac{\partial H_{n}}{\partial x_{p+j}}-i \frac{\partial H_{n}}{\partial y_{p+j}}\right\}, \\
\sum_{k=1}^{n-1} \beta_{k}=2, \quad \beta_{k}>0, \quad\left|\sigma_{k}\right|=1, \quad k, j=1, \ldots, n-1 .
\end{array}
$$

THeOREM 4. The parameters defining the function of the class $\Sigma_{a}$ extremal for the functional $E_{n}\left(F^{\prime}\right)$ satisfy the following system of equations:

$$
\begin{gathered}
B_{n+1} \sigma_{k}^{2(n+1)}+\ldots+B_{1} \sigma_{k}^{n+2}+\lambda \sigma_{k}^{n+1}+\bar{B}_{1} \sigma_{k}^{n}+\ldots+\bar{B}_{n+1}=0 \\
2(n+1) B_{n+1} \sigma_{k}^{2 n+1}+\ldots+(n+2) B_{1} \sigma_{k}^{n+1}+(n+1) \lambda \sigma_{k}^{n}+ \\
+n \bar{B}_{1} \sigma_{k}^{n-1}+\ldots+\bar{B}_{n}=0 \\
B_{j}=\frac{1}{1-a i} \sum_{p=-1}^{n-j} b_{p}\left\{\frac{\partial E_{n}}{\partial x_{p+j}}-i \frac{\partial E_{n}}{\partial y_{p+j}}\right\} \\
\sum_{k=1}^{n+1} \beta_{k}=2, \quad \beta_{k}>0, \quad\left|\sigma_{k}\right|=1, \quad k, j=1, \ldots, n+1 .
\end{gathered}
$$

The proof of theorem 3. The coefficients of function $f(z)$ of class $S_{a}$ extremal for functional $H_{n}(f)$ are given by formula $(7)$ where by theorem 1

$$
\alpha_{j}=\sum_{k=1}^{n-1} \beta_{k} \sigma_{k}^{j}, \quad \sum_{k=1}^{n-1} \beta_{k}=2, \quad \beta_{k}>0, \quad \sigma_{k}=e^{i \vartheta_{k}}
$$

So the coefficients are polynomials of variables $\beta_{k}$ and $\sigma_{k}$. Further we have

$$
\begin{aligned}
\frac{\partial f}{\partial \beta_{k}} & =-z\left[\prod_{j=1}^{n-1}\left(1-\sigma_{k} z\right)^{-\beta_{j}}\right]^{1 /(1-a i)} \log \left(1-\sigma_{k} z\right) \frac{1}{1-a i} \\
& =\frac{\partial a_{2}}{\partial \beta_{k}} z^{2}+\frac{\partial a_{3}}{\partial \beta_{k}} z^{3}+\ldots+\frac{\partial a_{p}}{\partial \beta_{k}} z^{p}+\ldots
\end{aligned}
$$

since

$$
\begin{aligned}
-z\left[\prod_{j=1}^{n-1}\left(1-\sigma_{h} z\right)^{-\beta_{j}}\right]^{1 /(1-a i)} \log \left(1-\sigma_{k} z\right) \frac{1}{1-a i} \\
=\frac{1}{1-a i} \sum_{p=2}^{\infty} z^{p}\left\{\frac{\sigma_{k}^{p-1}}{p-1}+\frac{\sigma_{k}^{p-2}}{p-2} a_{2}+\ldots+\sigma_{k} a_{p-1}\right\}
\end{aligned}
$$

we have

$$
\frac{\partial a_{p}}{\partial \beta_{k}}=-\frac{1}{1-a i}\left\{\frac{\sigma_{k}^{p-1}}{p-1}+\frac{\sigma_{k}^{p-2}}{p-2} a_{2}+\ldots+\sigma_{k} a_{p-1}\right\}
$$

Similarly

$$
\frac{\partial a_{p}}{\partial \beta_{k}}=-\frac{i \beta_{k}}{1-a i}\left\{\sigma_{k}^{p-1}+\sigma_{k}^{p-2} a_{2}+\ldots+\sigma_{k} a_{p-1}\right\}
$$

In order to determine the extremum of the functional $H_{n}(f)$, it is sufficient, to find the extremum of the function $H_{n}\left(x_{2}, \ldots, x_{n} ; y_{2}, \ldots, y_{n}\right)$ on the boundary of the region $W_{n}^{a}$. After replacing the variables $x_{k}, y_{k}$ by the variables $\beta_{k}$ and $\vartheta_{k}$ we have to find the extremum of the function $H_{n}\left(\vartheta_{1}, \ldots, \vartheta_{n-1} ; \beta_{1}, \ldots, \beta_{n-1}\right)$ with the condition $\sum_{k=1}^{n-1} \beta_{k}=2$ in the interval of variability $0 \leqslant \beta_{k} \leqslant 2,-\infty<\vartheta_{k}<\infty$. Let $\lambda^{\prime}$ be a Lagrange multiplier and let us find the derivatives of the function

$$
H_{n}^{*}=H_{n}+\lambda^{\prime}\left(\sum_{k=1}^{n-1} \beta_{k}-2\right)
$$

at the extremal point. We have

$$
\begin{aligned}
& \frac{\partial H_{n}^{*}}{\partial \beta_{k}}=\sum_{j=2}^{n} \frac{\partial H_{n}}{\partial x_{j}} \cdot \frac{\partial x_{j}}{\partial \beta_{k}}+\sum_{j=2}^{n} \frac{\partial H_{n}}{\partial y_{j}} \cdot \frac{\partial y_{j}}{\partial \beta_{k}}+\lambda^{\prime}=0, \\
& \frac{\partial H_{n}^{*}}{\partial \vartheta_{k}}=\sum_{j=2}^{n} \frac{\partial H_{n}}{\partial x_{j}} \cdot \frac{\partial x_{j}}{\partial \vartheta_{k}}+\sum_{j=2}^{n} \frac{\partial H_{n}}{\partial y_{j}} \cdot \frac{\partial y_{j}}{\partial \vartheta_{k}}=0 .
\end{aligned}
$$


Putting $x_{p}=\operatorname{re} a_{p}=\frac{1}{2}\left(a_{p}+\bar{a}_{p}\right), y_{p}=\operatorname{im} a_{p}=\frac{i}{2}\left(\bar{a}_{p}-a_{p}\right)$ and substituting (11) and (12) into (13) we obtain the following system of equations:

$$
\begin{aligned}
\frac{1}{1-a i} \sum_{j=1}^{n-1} \frac{\sigma_{k}^{j}}{j} \sum_{p=1}^{n-j} a_{p}\left\{\frac{\partial H_{n}}{\partial x_{p+j}}-i \frac{\partial H_{n}}{\partial y_{p+j}}\right\}+ \\
+\frac{1}{1+a i} \sum_{j=1}^{n-1} \frac{\bar{\sigma}_{k}^{j}}{j} \sum_{p=1}^{n-j} \bar{a}_{p}\left\{\frac{\partial H_{n}}{\partial x_{p+j}}+i \frac{\partial H_{n}}{\partial y_{p+j}}\right\}+\lambda^{\prime}=0
\end{aligned}
$$

$$
\begin{aligned}
\beta_{k}\left[\frac{1}{1-a i} \sum_{j=1}^{n-1} \sigma_{k}^{j}\right. & \sum_{p=1}^{n-j} a_{p}\left\{\frac{\partial H_{n}}{\partial x_{p+j}}-i \frac{\partial H_{n}}{\partial y_{p+j}}\right\}- \\
& \left.-\frac{1}{1+a i} \sum_{j=1}^{n-1} \bar{\sigma}_{k}^{j} \sum_{p=1}^{n-j} \bar{a}_{p}\left\{\frac{\partial H_{n}}{\partial x_{p+j}}+i \frac{\partial H_{n}}{\partial y_{p+j}}\right\}\right]=0, \\
\sum_{k=1}^{n-1} \beta_{k} & =2 .
\end{aligned}
$$

Without loss of generality we can assume that no $\beta_{k}$ are equal to 0 . Further we have $\bar{\sigma}_{k}=1 / \sigma_{k}$. To be short let us substitute

$$
A_{j}=\frac{1}{1-a i} \cdot \frac{1}{j} \sum_{p=1}^{n-j} a_{p}\left\{\frac{\partial H_{n}}{\partial x_{p+j}}-i \frac{\partial H_{n}}{\partial y_{p+j}}\right\} .
$$

By simple transformations we find from (14) that the parameters of the extremal function must satisfy the system of equations

$$
A_{n-1} \sigma_{k}^{2(n-1)}+\ldots+A_{1} \sigma_{k}^{n}+\lambda \sigma_{k}^{n-1}+\bar{A}_{1} \sigma_{k}^{n-2}+\ldots+\bar{A}_{n-1}=0,
$$

(15) $2(n-1) A_{n-1} \sigma_{k}^{2 n-3}+\ldots+n A_{1} \sigma_{k}^{n-1}+(n-1) \lambda \sigma_{k}^{n-2}+$

$$
\begin{aligned}
& +(n-2) \bar{A}_{1} \sigma_{k}^{n-3}+\ldots+\bar{A}_{n-2}=0, \\
& \sum_{k=1}^{n-1} \beta_{k}=2, \quad k=1, \ldots, n-1 .
\end{aligned}
$$

where it is not necessary that all $\sigma_{k}$ should be different. The proof of theorem 4 is analogical.

Putting $a=0$ we obtain from theorems 3 and 4 the wellknown theorems on starlike functions (see [4]).

THEOREM 5. The coefficients of the class $\Sigma_{a}$ satisfy the inequality

$$
\left|b_{n}\right| \leqslant \frac{2}{n+1} \cdot \frac{1}{\sqrt{1+a^{2}}}
$$

We obtain an equality for the function

$$
F(z)=\frac{1}{z}\left[\left(1+\eta z^{n+1}\right)^{2 /(n+1)}\right]^{1 /(1-a i)}, \quad|\eta|=1 .
$$

Proof of theorem 5. J. Clunie in his paper [2] proves this for the class of starlike function with a pole. His method may easily be applied to a function of the class $\Sigma_{a}$. Namely

$$
F(z)=\frac{1}{z} \exp \left\{-\varrho \int_{0}^{z} \frac{p(z)-1}{z} d z\right\}
$$

where

$$
\varrho=\frac{1}{1-a i}, \quad \operatorname{re} p(z) \geqslant 0, \quad p(z)=1+\alpha_{1} z+\ldots
$$

Hence

$$
\frac{z F^{\prime}(z)}{F(z)}=-\varrho[p(z)-1]-1
$$

Let

$$
\omega(z)=\frac{p(z)-1}{p(z)+1}=\sum_{k=1}^{\infty} \omega_{k} z^{k} .
$$

Then $|\omega(z)| \leqslant 1$. From (16) and (17) we have

or

$$
\omega(z)\left[z F^{\prime}(z)-(2 \varrho-1) F(z)\right]=z F^{\prime}(z)+F(z)
$$

(18) $\sum_{k=1}^{\infty} \omega_{k} z^{k}\left\{-2 \varrho+\sum_{k=0}^{\infty}(k+1-2 \varrho) b_{k} z^{k+1}\right\}=\sum_{k=0}^{\infty}(k+1) b_{k} z^{k+1}$.

Hence we have

$$
\begin{aligned}
\sum_{k=1}^{\infty} \omega_{k} z^{k}\left\{-2 \varrho+\sum_{k=0}^{n-1}(k+1-2 \varrho) b_{k} z^{k+1}\right\} & \\
& =\sum_{k=0}^{n}(k+1) b_{k} z^{k+1}+\sum_{k=n+2}^{\infty} c_{k} z^{n}
\end{aligned}
$$

$c_{k}$ being some complex numbers. Since $|\omega(z)| \leqslant 1$, we have

$$
\sum_{k=0}^{n}(k+1)^{2}\left|b_{k}\right|^{2} r^{2(k+1)}+\sum_{k=n+2}^{\infty}\left|c_{k}\right|^{2} r^{2 k} \leqslant 4|\varrho|^{2}+\sum_{k=0}^{n-1}|k+1-2 \varrho|^{2}\left|b_{k}\right|^{2} r^{2 k}
$$


for $r<1$. Hence further

$$
\sum_{k=0}^{n}(k+1)^{2}\left|b_{k}\right|^{2} \leqslant 4|\varrho|^{2}+\sum_{k=0}^{n-1}|k+1-2 \varrho|^{2}\left|b_{k}\right|^{2}
$$

or

$$
(n+1)^{2}\left|b_{n}\right|^{2} \leqslant 4|\varrho|^{2}-4|\varrho|^{2} \sum_{k=1}^{n-1} k\left|b_{k}\right|^{2}
$$

and thus

$$
\left|b_{n}\right| \leqslant \frac{2}{n+1} \cdot \frac{1}{\sqrt{1+a^{2}}} .
$$

We obtain an equality for the coefficients of the function

$$
F(z)=\frac{1}{z}\left[\left(1+\eta z^{n+1}\right)^{2 /(n+1)}\right]^{1 /(1-a i)}, \quad|\eta|=1 .
$$

THEOREM 6 . The coefficients of a function of class $S_{a}$ satisfy the inequality

$$
\left|a_{n}\right| \leqslant \frac{\left(1+a^{2}\right)^{(1-n) / 2}}{(n-1) !} \prod_{k=0}^{n-2}\left[(2+k)^{2}+k^{2} a^{2}\right]^{1 / 2} .
$$

The sign of equality occurs only for the coefficients of the function

$$
f(z)=z(1+\eta z)^{-2 /(1-a i)}, \quad|\eta|=1 .
$$

Proof of theorem 6. Just as in the proof of theorem 5, the function of class $S_{a}$

$$
f(z)=z \exp \left\{\varrho \int_{0}^{z} \frac{p(z)-1}{z} d z\right\}
$$

satisfies the equation

$$
\frac{z f^{\prime}(z)}{f(z)}=\varrho[p(z)-1]+1
$$

Putting the bounded function $\omega(z)$ we obtain from (20) the equation

$$
\omega(z)\left[z f^{\prime}(z)+(2 \varrho-1) f(z)\right]=z f^{\prime}(z)-f(z)
$$

or otherwise

$$
\sum_{k=1}^{\infty} \omega_{k} z^{k} \sum_{k=1}^{\infty}(k+2 \varrho-1) a_{k} z^{k}=\sum_{k=1}^{\infty}(k-1) a_{k} z^{k}
$$

Hence of course

$$
\sum_{k=1}^{\infty} \omega_{k} z^{k} \sum_{k=1}^{n-1}(k+2 \varrho-1) a_{k} z^{k}=\sum_{k=1}^{n}(k-1) a_{k} z^{k}+\sum_{k=n+1}^{\infty} c_{k} z^{k}
$$

where $c_{k}$ are some numbers. Since $|\omega(z)| \leqslant 1$, we have

$$
\sum_{k=1}^{n}(k-1)^{2}\left|a_{k}\right|^{2} r^{2 k}+\sum_{k=n+1}^{\infty}\left|c_{k}\right|^{2} r^{2 k} \leqslant \sum_{k=1}^{n-1}|k+2 \varrho-1|^{2}\left|a_{k}\right|^{2} r^{2 k}
$$

for $r<1$. Hence

$$
\sum_{k=1}^{n}(k-1)^{2}\left|a_{k}\right|^{2} \leqslant \sum_{k=1}^{n-1}|k+2 \varrho-1|^{2}\left|a_{k}\right|^{2}
$$

$$
\left|a_{n}\right|^{2} \leqslant \frac{4}{(n-1)^{2}} \sum_{k=1}^{n-1} \frac{k}{1+a^{2}}\left|a_{k}\right|^{2}
$$

For $n=2$ we have

$$
\left|a_{2}\right| \leqslant \frac{2}{\sqrt{1+a^{2}}} .
$$

Hence and from formula (23) by simple induction we conclude that

$$
\left|a_{n}\right| \leqslant \frac{\left(1+a^{2}\right)^{(1-n) / 2}}{(n-1) !} \prod_{k=0}^{n-2}\left[(2+k)^{2}+k^{2} a^{2}\right]^{1 / 2}
$$

We obtain an equality for the coefficients of the function

$$
f(z)=z(1+\eta z)^{-2 /(1-\alpha i)}, \quad|\eta|=1 .
$$

\section{References}

[1] C. Carathéodory, Über den Variabilitätsbereich der Fourierschen Konstanten von positiven harmonischen Funktionen, Rend. Circ. Mat. Palermo 32 (1911), p. 193 - 217.

[2] J. Clunie, On meromorphio sehlioht functions, Journ. London Math. Soo. 34 (1959), p. $115-116$.

[3] L. Špaček, Př́spěvek to teorii funkci prostych, Časopis Pěst. Mat. 62 (1933), p. $12-19$.

[4] J. Zamorski, Equations satisfied by the extremal starlike functions, Ann. Polon. Math. 5 (1958), p. $285-291$. INSTYTUT MATEMATYCZNY POLSKIEJ AKADEMII NAUK
MATHEMATICAL INSTITUTE OF THE POLISH ACADEMY OF SCIENCES

Reģu par la Rédaction le 29. 10. 1959 